\title{
Epicardial Adipose Tissue Thickness and Its Association With the Presence and Severity of Coronary Artery Disease in Clinical Setting: A Cross-Sectional Observational Study
}

\author{
Santosh Kumar Sinha ${ }^{\mathrm{a}, \mathrm{b}}$, Ramesh Thakur ${ }^{\mathrm{a}}$, Mukesh Jitendra Jha ${ }^{\mathrm{a}}$, Amit Goel ${ }^{\mathrm{a}}$, Varun Kumara ${ }^{\mathrm{a}}$, \\ Ashutosh Kumar ${ }^{a}$, Vikas Mishra ${ }^{a}$, Chandra Mohan Varma ${ }^{a}$, Vinay Krishna ${ }^{a}$, Avinash Kumar Singha, \\ Mohit Sachan ${ }^{\mathrm{a}}$
}

\begin{abstract}
Background: Obesity is an important risk factor for atherosclerotic cardiovascular disease (ASCVD). Estimation of visceral adipose tissue is important and several methods are available as its surrogate. Although correlation of epicardial adipose tissue (EAT) with visceral adipose tissue as estimated by magnetic resonance imaging (MRI) and/or CT is excellent, it is costlier and cumbersome. EAT can be accurately measured by two-dimensional (2D) echocardiography. It tends to be higher in patients with acute coronary syndrome than in subjects without coronary artery disease (CAD) and in those with stable angina. It also carries advantage as index of high cardiometabolic risk as it is a direct measure of visceral fat rather than anthropometric measurements. The present study evaluated the relationship of EAT to the presence and severity of CAD in clinical setting.
\end{abstract}

Methods: In this prospective, single-center study conducted in the Department of Cardiology, LPS Institute of Cardiology, Kanpur, India, 549 consecutive patients with acute coronary syndrome or chronic stable angina were enrolled. Sensitivity, specificity, and receiver operating characteristic (ROC) curve were estimated to find cut-off value of EAT thickness for diagnosing $\mathrm{CAD}$ using coronary angiographic findings as gold standard.

Results: Patients were diagnosed as CAD group $(n=464,60.30 \pm$ 8.36 years $)$ and non-CAD group $(n=85,54.42 \pm 11.93$ years $)$ after assessing coronary angiograms. The EAT was measured at end-systole from the PLAX views of three cardiac cycles on the free wall of the right ventricle. Lesion was significant if $>50 \%$ in left main and $>70 \%$ in other coronary arteries. The mean EAT thickness in CAD group was $5.10 \pm 1.06$ and in non-CAD group was $4.36 \pm 1.01$ which was significantly $(\mathrm{P}=0.003)$. Significant correlation was demonstrated between EAT thickness and presence of CAD $(\mathrm{P}<0.003)$. Higher EAT was associated with severe $\mathrm{CAD}$ and presence of multivessel disease. By ROC analysis, EAT $>4.65 \mathrm{~mm}$ predicated the presence of sig-

Manuscript accepted for publication January 25, 2016

aDepartment of Cardiology, LPS Institute of Cardiology, G.S.V.M. Medical College, Kanpur, Uttar Pradesh 208002, India

${ }^{b}$ Corresponding Author: Santosh Kumar Sinha, Department of Cardiology, LPS Institute of Cardiology, G.S.V.M. Medical College, Kanpur, Uttar Pradesh 208002, India. Email: fionasan@rediffmail.com

doi: http://dx.doi.org/10.14740/jocmr2468w nificant coronary stenosis by $71.6 \%$ sensitivity and $73.1 \%$ specificity.

Conclusion: EAT thickness measured using transthoracic echocardiography (TTE) significantly correlates with the presence and severity of CAD. It is sensitive, easily available, and cost-effective and assists in the risk stratification and may be an additional marker on classical risk factors for CAD.

Keywords: Acute coronary syndrome; Chronic stable angina; Coronary artery stenosis; Epicardial fat thickness; Echocardiography; ROC curve; TTE

\section{Introduction}

Obesity is recognized as an important risk factor for the development of all features of metabolic syndrome and atherosclerotic cardiovascular disease (ASCVD) [1-6]. Epicardial adipose tissue (EAT) is true visceral fat deposit which is commonly found in the atrioventricular and interventricular grooves, interacting locally and modulating the coronary arteries through the paracrine or vasocrine secretion of proinflammatory adipokines. Because EAT shares a common coronary blood supply with the myocardium [7], it has been proposed that EAT is able to synthesize and secrete adipokines and bioactive factors that reach the myocardium through vasocrine or paracrine pathways [8-10]. There is an excellent correlation of EAT with visceral adipose tissue as estimated by magnetic resonance imaging (MRI). EAT significantly correlates with the extent and severity of coronary artery disease (CAD), as assessed by coronary angiography. However, the identification of abundant amounts of EAT in patients clinically referred for cardiac imaging may raise concerns about cardiometabolic conditions of the patient. Epicardial adipocytes are anatomically, morphologically and functionally different from other fat depots. Under physiological and metabolically high-demand conditions, it supplies energy and heat to the myocardium and exerts a protective role. Its pathological increase and the cooccurrence of other metabolic and hemodynamic abnormalities turn it into an adverse lipotoxic, prothrombotic and proinflammatory organ supporting a dichotomous role $[11,12]$.

Visceral fat is typically measured by surrogate markers, such as waist circumference or the ratio of waist circumfer- 


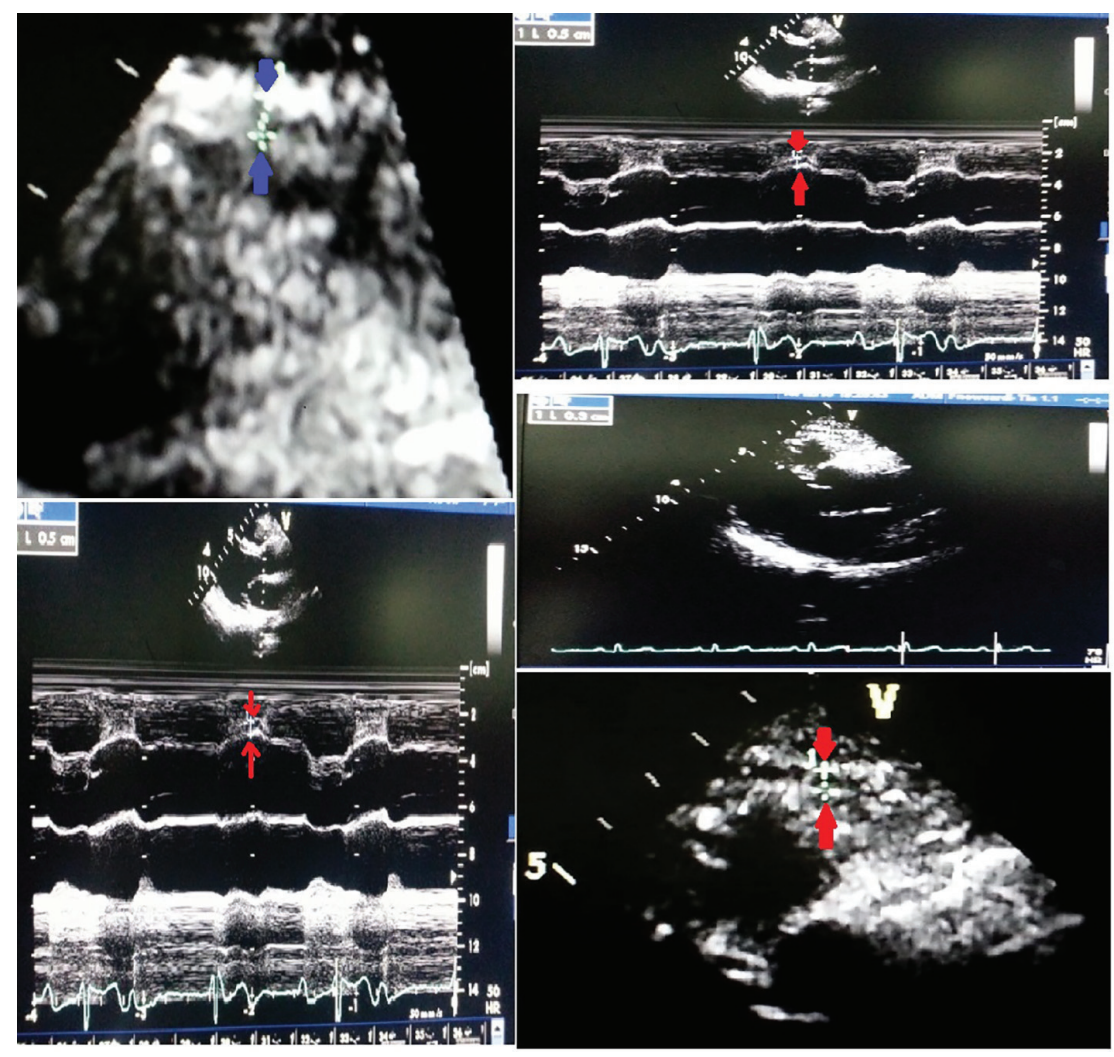

Figure 1. EAT thickness is identified as the echo-free space between the outer wall of the myocardium and the visceral layer of pericardium in the PLAX view.

ence to hip circumference and its direct measurement including MRI and/or CT is expensive and cumbersome. So there is a compelling need and growing interest in less expensive and more reliable imaging markers of visceral adiposity. Epicardial fat thickness can be visualized and measured with two-dimensional (2D) echocardiography as first proposed and validated by Iacobellis et al which appears as the relatively echo-free space between the outer wall of the myocardium and the visceral layer of pericardium $[13,14]$.

\section{Materials and Methods}

\section{Patients}

The aim of the study was to assess the association between epicardial fat thickness, measured using transthoracic echocardiography (TTE), and the severity of coronary artery stenosis, measured using coronary angiography. The present study was a prospective, single-center trial conducted in the Department of Cardiology, LPS Institute of Cardiology, G.S.V.M. Medical College, Kanpur, UP, India between August 2014 and October 2015 spanning over 15 months. Five hundred forty-five consecutive patients were enrolled who presented with acute coronary syndrome or chronic stable angina (CSA) and subsequently underwent complete evaluation including coronary angiography. Exclusion criteria were patients with prior history of documented acute coronary syndrome, coronary artery bypass surgery or percutaneous coronary intervention prior to the current hospitalization, undergoing primary PCI and whose TTE imaging was inadequate for the measurement of epicardial fat thickness. Informed consent was obtained from each patient enrolled in the study.

Table 1. Baseline Characteristics of Patients: Age and Sex Distribution

\begin{tabular}{llllll}
\hline Age group & Male $(\mathbf{n}=\mathbf{4 0 5})$ & $\mathbf{\%}$ & Female $(\mathbf{n}=\mathbf{1 4 4})$ & $\mathbf{\%}$ & Total \\
\hline $20-30$ years & 24 & 6 & 5 & 3 & 29 \\
$30-40$ years & 56 & 14 & 33 & 23 & 89 \\
$40-50$ years & 118 & 29 & 29 & 20 & 147 \\
$50-60$ years & 136 & 33 & 46 & 32 & 182 \\
$>60$ years & 71 & 18 & 31 & 22 & 102 \\
\hline
\end{tabular}


Table 2. Clinical Diagnosis and Distribution of Patients

\begin{tabular}{lllll} 
Diagnosis & No. $(\mathbf{n})$ & Male (n) & Female (n) & $\mathbf{\%}$ \\
\hline CSA & 115 & 95 & 20 & 21 \\
USA & 81 & 44 & 37 & 15 \\
NSTEMI & 153 & 95 & 58 & 28 \\
STEMI & 200 & 171 & 29 & 36 \\
Total & 549 & 405 & 144 & 100 \\
\hline
\end{tabular}

\section{Data collection}

Enrolled patients underwent complete evaluation including detailed clinical examination and investigations including ECG, cardiac enzymes, 2D echocardiography and coronary angiogram as a part of their diagnostic procedure to identify the involved coronary artery. The 12-lead ECG was recorded for all patients at a speed of $25 \mathrm{~mm} / \mathrm{s}$ and voltage of $10 \mathrm{~mm} / \mathrm{mV}$ on a 12-channel Mortara ELI 250 ECG machine. ST-segment deviation was measured at $60 \mathrm{~ms}$ from point $\mathrm{J}$ in all leads. Echocardiograms were performed with a VIVID7 (GE) instrument. Epicardial fat thickness was measured in standard parasternal long-axis (PLAX) view. It was defined as the relatively echo-free space between the outer wall of the myocardium and the visceral layer of pericardium; its thickness was measured perpendicularly on the free wall of the right ventricle at end-systole in three cardiac cycles as it was compressed during diastole and average was taken using aortic annulus as anatomical reference (Fig. 1).

Coronary angiography was performed during indexed period using the Judkins' method after proper consent by radial artery approach using Tiger catheter (Terumo Inc, Japan). Hemodynamically, significant stenosis was defined as a diameter stenosis of $\geq 50 \%$ in left main coronary artery (LMCA) and $\geq 70 \%$ in vessels other than LMCA and non-obstructive CAD was defined $\leq 50 \%$. Subjects were divided into two groups: CAD group and non-CAD group.

\section{Statistical analysis}

Continuous variables were expressed as mean $\pm \mathrm{SD}$, while discrete variables were presented as frequency and percentage. The $\chi^{2}$ test was used to compare categorical variables and unpaired $t$-test was used to compare continuous variables between $\mathrm{CAD}$ and non-CAD patients. The relative risk (RR) with its $95 \%$ confidence interval (CI) was calculated to find the strength of association. The receiver operating characteristic (ROC) curve was drawn to find the cut-off value of epicardial fat thickness for diagnosing CAD. The sensitivity and specificity were calculated. The P-value $<0.05$ was considered significant. All the analysis was carried out by using STATA 8.0 version (Chicago, IL, USA).

\section{Results}

\section{Baseline characteristics}

The baseline characteristics of enrolled patients are described in Table 1. Age stratification revealed that more than one-third of patients were between 50 and 60 years $(33 \%)$, followed by 40 - 50 years $(27 \%),>60$ years $(18 \%), 30$ - 40 years $(16 \%)$, and 20 - 30 years $(5 \%)$. Furthermore, majority of enrolled patients were males (73\%). ST-segment elevation myocardial infarction (STEMI) was the commonest presentation $(n=200 ; 36 \%)$ followed by non-ST-segment elevation myocardial infarction (NSTEMI: $\mathrm{n}=153 ; 28 \%)$, CSA $(\mathrm{n}=115 ; 21 \%)$, and unstable angina (USA: $\mathrm{n}=81 ; 15 \%$ ) in Table 2 and Figure 2. Anterior wall myocardial infarction (AWMI) was the commonest presentation with 138 patients, while 62 had inferior wall myocardial infarction (IWMI). Most of the patients in this study of any diagnosis were in $>50$ years age group. Most of the non-CAD was observed among USA followed by CSA and NSTEMI group and none in STEMI group and comparison of presenting symptoms between CAD and non-CAD cases is shown in Table 3.

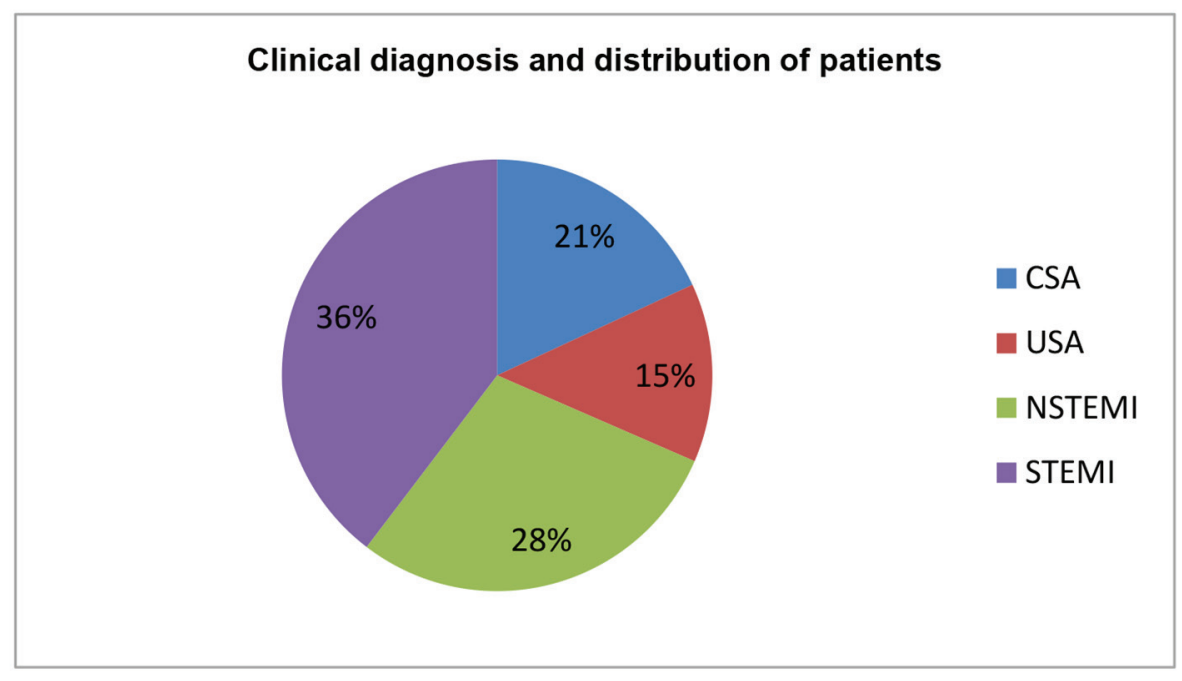

Figure 2. Clinical diagnosis and distribution of patients. 
Table 3. Comparison of Presenting Symptoms Between CAD and Non-CAD Cases

\begin{tabular}{|c|c|c|c|c|c|c|}
\hline \multirow{2}{*}{ Diagnosis } & \multicolumn{2}{|c|}{$\operatorname{CAD}(n=464)$} & \multicolumn{2}{|c|}{ Non-CAD $(n=85)$} & \multirow{2}{*}{ RR (95\% CI) } & \multirow{2}{*}{ P-value } \\
\hline & No. & $\%$ & No. & $\%$ & & \\
\hline CSA & 96 & 20 & 19 & 22 & $0.92(0.71-1.18)$ & 0.51 \\
\hline USA & 31 & 7 & 50 & 59 & $0.89(0.55-0.43)$ & 0.59 \\
\hline NSTEMI & 137 & 29 & 16 & 19 & $0.97(0.73-0.30)$ & 0.87 \\
\hline STEMI & 200 & 44 & 0 & 0 & $1.15(0.91-0.44)$ & 0.25 \\
\hline Total & 464 & 100 & 85 & 100 & & \\
\hline
\end{tabular}

RR: relative risk; Cl: confidence interval.

Table 4. Epicardial Adipose Tissue Thickness Among Cases With Relation to Age

\begin{tabular}{|c|c|c|c|c|c|}
\hline Age group & EAT $2 \mathrm{~mm}$ & EAT 3 - 4 mm & EAT 4 - 5 mm & EAT 5 - 6 mm & EAT $>6 \mathrm{~mm}$ \\
\hline $20-30$ years & 2 & 8 & 9 & 6 & 4 \\
\hline $30-40$ years & 17 & 21 & 15 & 18 & 18 \\
\hline $40-50$ years & 8 & 55 & 42 & 31 & 11 \\
\hline $50-60$ years & 2 & 43 & 32 & 56 & 49 \\
\hline$>60$ years & 4 & 21 & 24 & 27 & 26 \\
\hline Total & 33 & 148 & 122 & 138 & 108 \\
\hline
\end{tabular}

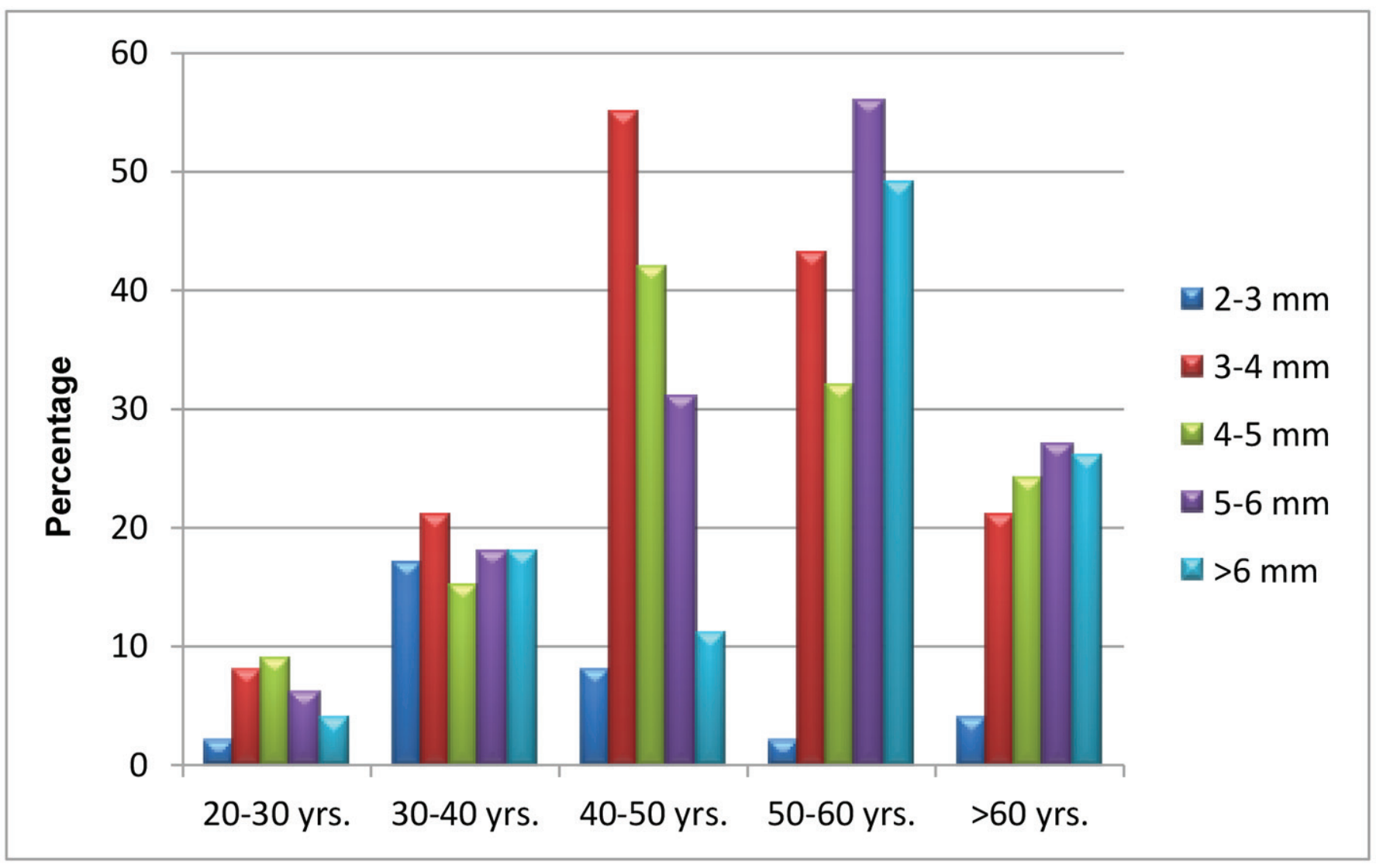

Figure 3. EAT thickness among cases with relation to sex (A) and age (B). 
Table 5. Comparison of Epicardial Fat Thickness Between CAD and Non-CAD and EAT With Relation to Clinical Diagnosis

\begin{tabular}{ll}
\hline Category & Epicardial fat thickness \\
\hline CAD & $5.16 \pm 1.06$ \\
Non-CAD & $4.23 \pm 1.01$ \\
Unpaired $t$-test (P-value) & $0.003^{*}$ \\
\hline
\end{tabular}

\section{EAT thickness among cases with relation to age, sex and diagnosis}

In a total of 549 patients, EAT of $3-4 \mathrm{~mm}$ was observed in maximum number of patients, i.e., 148 patients followed by $5-6 \mathrm{~mm}$ in 138 patients, while $4-5 \mathrm{~mm}$ was seen in 122 patients and remainder the rest (Table 4). Their sex distribution is depicted in Figure 3.

CAD was observed among 464 patients. Twenty patients had EAT in between 2 and $3 \mathrm{~mm}$, out of whom CSA, USA, NSTEMI, and STEMI comprised 5, 3, 8, and 4, respectively. One hundred fourteen patients had EAT in between 3 and 4 $\mathrm{mm}$, out of whom CSA, USA, NSTEMI, and STEMI comprised 21, 6, 34, and 53, respectively. One hundred nineteen patients had EAT in between 4 and $5 \mathrm{~mm}$, out of whom CSA, USA, NSTEMI, and STEMI comprised 31, 7, 39, and 42, respectively. One hundred thirteen patients had EAT in between 5 and $6 \mathrm{~mm}$, out of whom CSA, USA, NSTEMI, and STEMI comprised $13,8,35$, and 57 , respectively. Ninety-eight patients had EAT of $>6 \mathrm{~mm}$, out of whom CSA, USA, NSTEMI, and
STEMI comprised 26, 7, 21, and 44, respectively. The average epicardial fat thickness was $5.16 \pm 1.06$ in CAD patients compared to $4.33 \pm 1.01$ in non-CAD and was significantly higher $(\mathrm{P}=0.003)$ (Table 5 and Fig. 4). In a total of 464 patients, 26 patients had non-obstructive CAD $(<50 \%)$. Significant CAD defined as $>70 \%$ in $\geq 1$ major epicardial artery and $>50 \%$ in LMCA was seen in 438 patients. Fifty-five patients had significant left main disease with or without other vessels disease. Out of these 55 patients, 12 were of CSA, eight were of USA, 19 were of NSTEMI, and 16 were of STEMI. Two hundred sixty-one patients had significant left anterior descending artery (LAD) disease with or without other vessels disease, of whom CSA, USA, NSTEMI, and STEMI were seen in 41, 11,73 , and 136, respectively. Sixty-seven patients with significant left circumflex artery (LCX) disease presented with CSA, USA, NSTEMI, and STEMI of 14, 5, 20, and 28, respectively. Thirty-two patients had significant right coronary artery (RCA) disease with or without other vessels disease. Out of these 32 patients, nine were of CSA, three were of USA, 11 were of NSTEMI, and nine were of STEMI. Eleven patients had significant Ramus disease with or without other vessels disease. Out of these 11 patients, four were of CSA, no patients were of USA, three were of NSTEMI, and four were of STEMI (Table 6 and Fig. 5).

\section{EAT thickness with relation to coronary angiogram and severity of CAD}

When CAD was further stratified according to EAT, signifi-

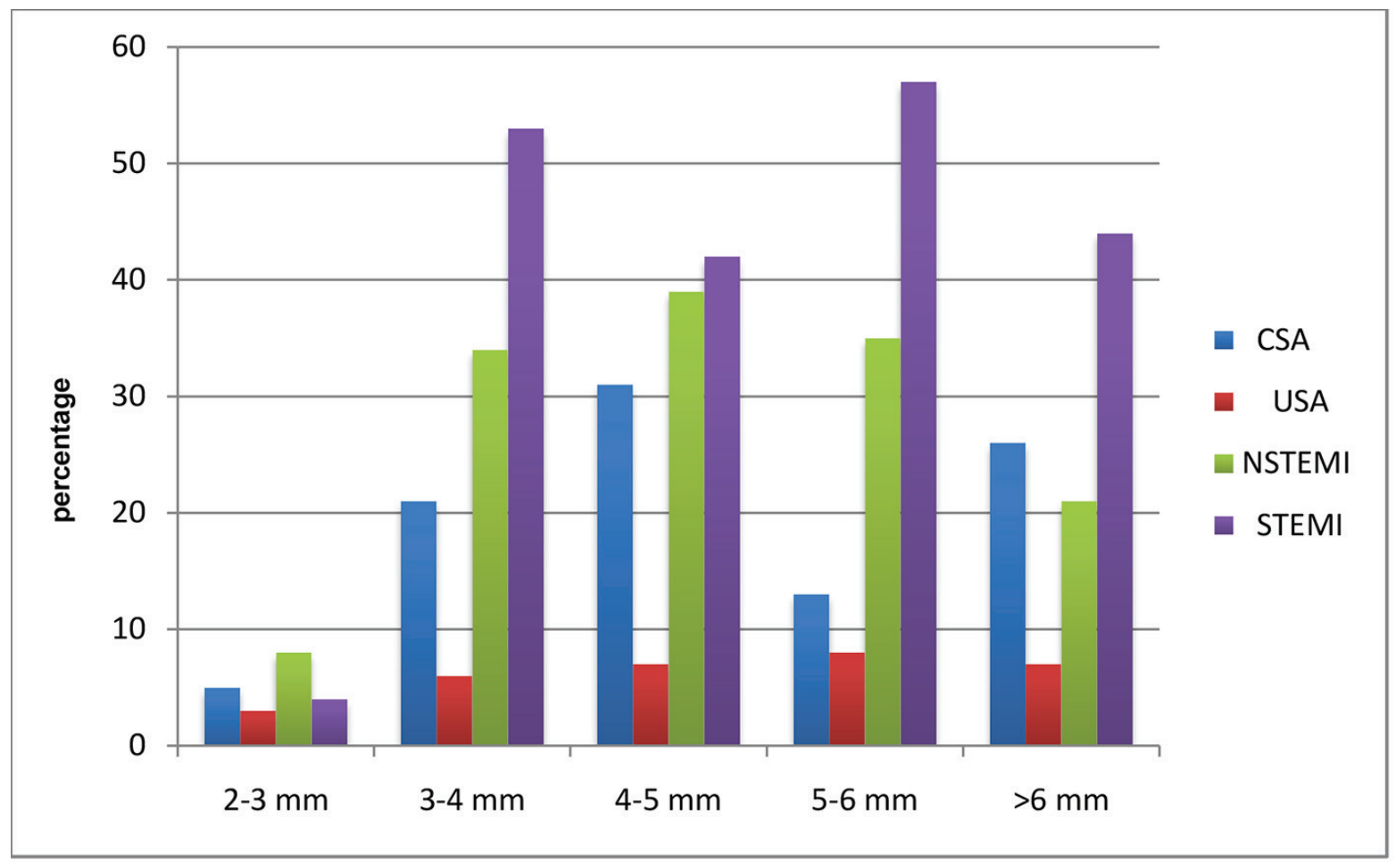

Figure 4. Comparison of epicardial fat thickness between CAD and non-CAD and EAT with relation to clinical diagnosis. 
Table 6. Clinical Presentation With Relation to Their Age

\begin{tabular}{|c|c|c|c|c|c|c|}
\hline Age group & LMCA (> 50\%) & LAD $(>70 \%)$ & $\operatorname{LCX}(>70 \%)$ & RCA $(>70 \%)$ & RI $(>70 \%)$ & Non-obstructive CAD \\
\hline 20 - 30 years & 1 & 45 & 15 & 29 & 5 & 1 \\
\hline $30-40$ years & 8 & 38 & 9 & 22 & 3 & 4 \\
\hline $50-60$ years & 12 & 49 & 18 & 33 & 6 & 8 \\
\hline$>60$ years & 24 & 73 & 13 & 23 & 7 & 6 \\
\hline
\end{tabular}

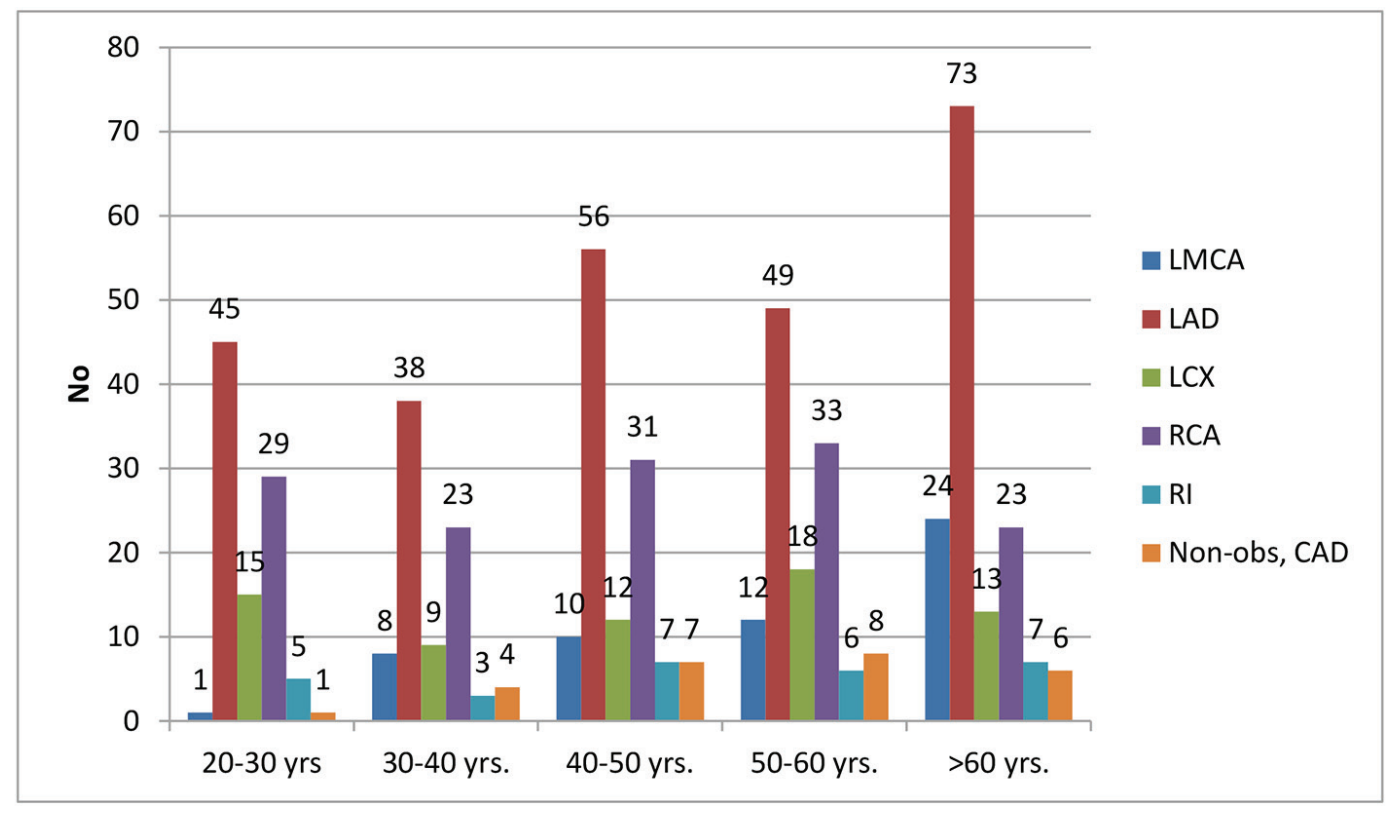

Figure 5. Clinical presentation with relation to their age.

cant left main was involved in 55 patients, of whom $0,5,9$, 15 , and 26 had EAT of $2-3,3-4,4-5,5-6$, and $>6 \mathrm{~mm}$, respectively. Two hundred sixty-one patients had significant LAD disease with or without other vessels disease, of whom $8,37,44,84$, and 88 had EAT of 2 - 3, 3- 4, 4 - 5, 5 - 6, and $>$ $6 \mathrm{~mm}$, respectively. Sixty-seven patients had significant LCX disease with or without other vessels disease, of whom 4, 7, 12, 19, and 25 had EAT of $2-3,3-4,4-5,5-6$, and $>6 \mathrm{~mm}$, respectively. RCA was involved in 138 patients, of whom 10 , $18,30,41$, and 39 patients had EAT of $2-3,3-4,4-5,5-6$, and $>6 \mathrm{~mm}$, respectively. Ramus intermedius was involved in 28 patients, of whom $3,5,9,4$, and 7 patients had EAT of $2-3$, $3-4,4-5,5-6$, and $>6 \mathrm{~mm}$, respectively (Table 7 and Fig. $6,7)$. In our observation among CAD patients, LAD involvement was commonest (57\%) as STEMI was the commonest presentation of ACS; RCA/PDA was culprit in 30\% of cases, whereas LCX/OM disease was observed in 14\% followed by LMCA disease in $11 \%$. RI disease was the least common as in $6 \%$ (Fig. 8). Epicardial fat thickness was $>4.65 \mathrm{~mm}$ in CAD patients with sensitivity and specificity of $71.6 \%$ and $73.1 \%$,

Table 7. Epicardial Adipose Tissue Thickness With Relation to Severity of CAD

\begin{tabular}{lllllll}
\hline EAT & LMCA & LAD & LCX & RCA & RI & Non-obstructive CAD \\
\hline $2-3 \mathrm{~mm}$ & 0 & 8 & 4 & 10 & 3 & 3 \\
$3-4 \mathrm{~mm}$ & 5 & 37 & 7 & 18 & 5 & 8 \\
$4-5 \mathrm{~mm}$ & 9 & 44 & 12 & 30 & 9 & 10 \\
$5-6 \mathrm{~mm}$ & 15 & 84 & 19 & 41 & 4 & 2 \\
$>6 \mathrm{~mm}$ & 26 & 88 & 25 & 39 & 7 & 3 \\
Total & 55 & 261 & 67 & 138 & 28 & 26 \\
\hline
\end{tabular}




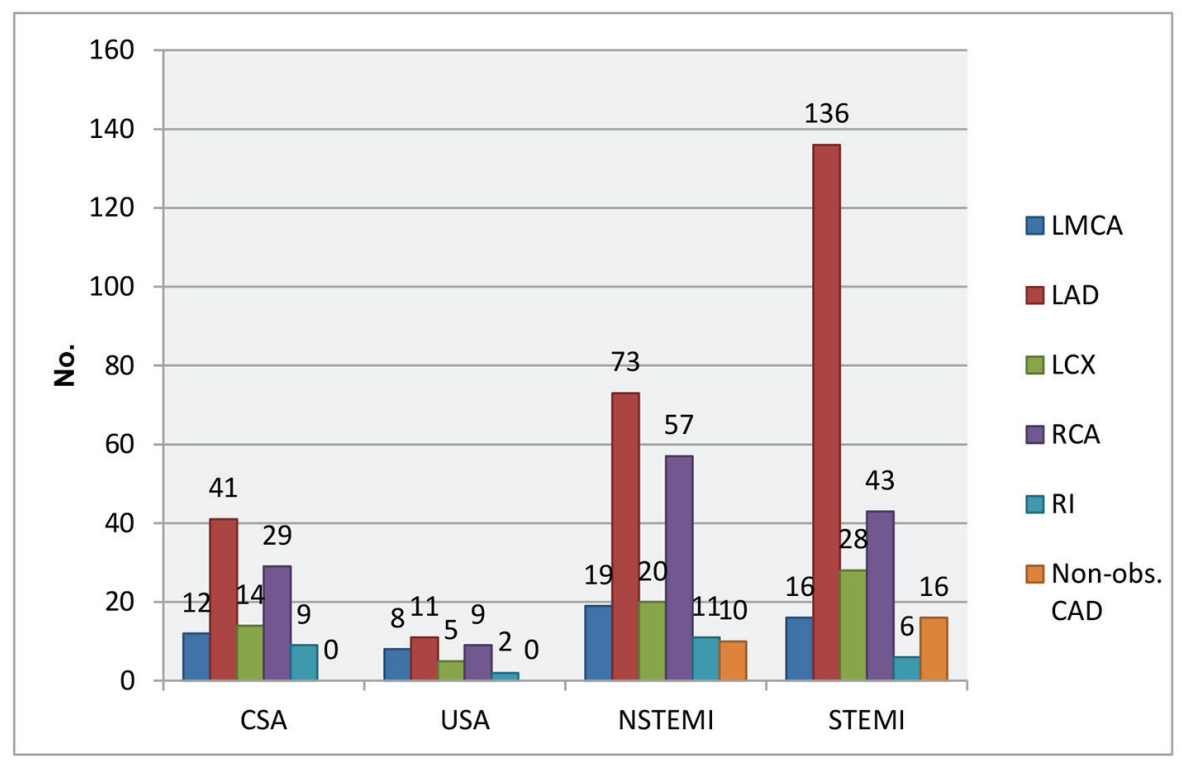

Figure 6. Epicardial adipose tissue thickness with relation to severity of CAD.

respectively (Table 8 ). The area under the curve on ROC curve analysis was $0.07(95 \% \mathrm{CI}: 0.58-0.82)$ which was statistically significant $(\mathrm{P}=0.002)$ (Fig. 9).

\section{Discussion}

Obesity is recognized as an important risk factor for the development of all features of metabolic syndrome and ASCVD. An estimation of visceral adipose tissue is important, and several methods are applied as a surrogate for the assessment of body composition and visceral adipose tissue. The relationship be- tween echocardiographic EAT and CAD has also been reported in previous study [15]. The majority of population-based clinical studies have reported excellent interobserver and intraobserver agreement for EAT thickness measurement. The present study evaluated the relationship of echocardiographic epicardial fat to the presence and severity of CAD in a clinical setting [16].

Jeong et al [17] showed the relationship between echocardiographic EAT and CAD in 203 patients who underwent echocardiography and coronary angiography. Coronary angiograms were analyzed for the extent and severity of CAD using Gensini score. The patients with a higher epicardial fat thickness were associated with a high Gensini score $(\mathrm{P}=0.014)$.

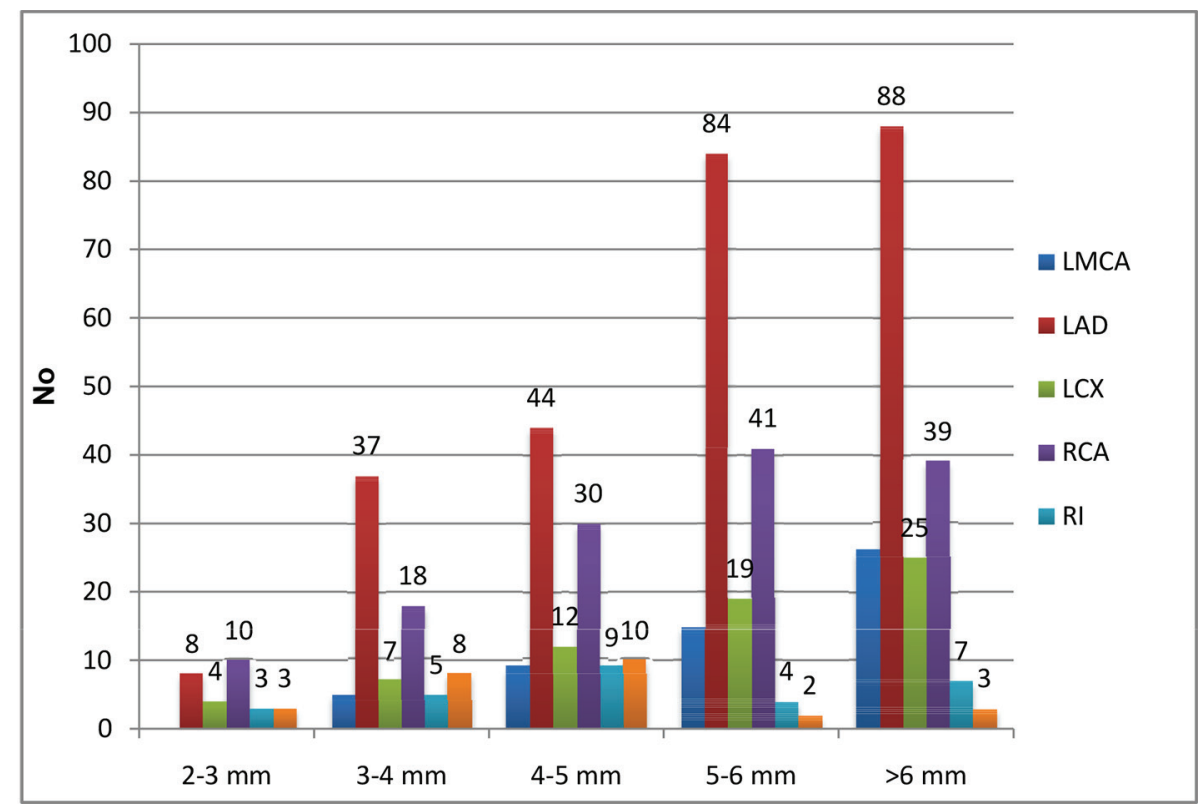

Figure 7. Angiographic findings depending on multiple responses. 


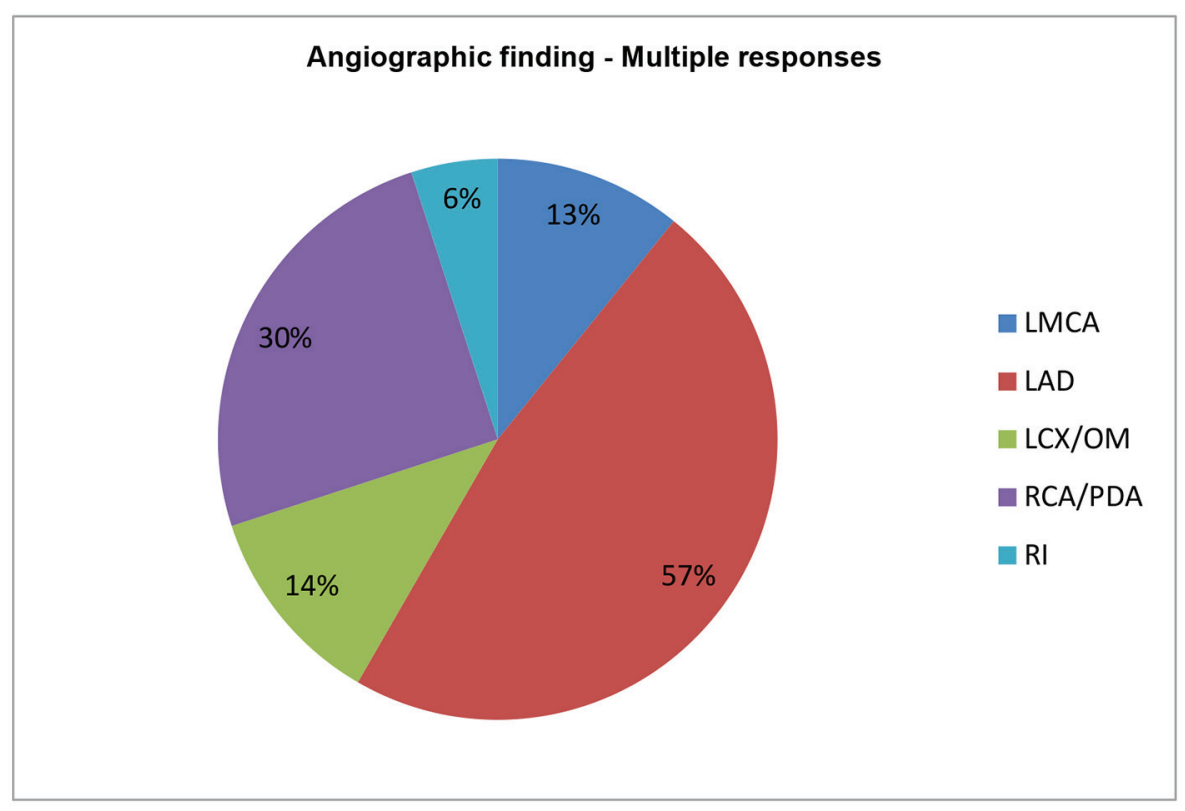

Figure 8. Angiographic findings depending on multiple responses.

Multivariate analysis showed that age (odds ratio (OR): 5.29, $\mathrm{P}=0.003)$, epicardial fat thickness (OR: 10.53, $\mathrm{P}=0.004)$, diabetes (OR: 8.06, $\mathrm{P}=0.006$ ), and smoking (OR: 14.65, $\mathrm{P}=$ 0.015 ) were independent factors affecting significant coronary artery stenosis. Interestingly, EAT was significantly correlated with the extent and severity of CAD, as assessed by the Gensini score. Contrary to Gensini score, we used visual estimation of plaque in coronary angiogram $(>50 \%$ in $\mathrm{LMCA},>70 \%$ in other coronary vessels) for the assessment of CAD.

Until now, MRI has been accepted as a gold standard for measuring epicardial fat thickness. Iacobellis et al reported the development of the echocardiographic measurement of epicardial fat $[11-13,18,19]$. They showed that echocardiographic epicardial fat thickness has a good correlation with MRI abdominal fat and epicardial fat measurements, and anthropometric and metabolic parameters. However, there have been few reports of the correlation between EAT and the presence and severity of CAD in a clinical setting. Chaowalit et al performed clinical study to confirm the association in 139 patients; however, they failed to demonstrate the association with the severity of CAD [20]. Our results also established that epicardial fat thickness has a good association with the severity of coronary artery stenosis.

Table 8. ROC Curve Analysis of Epicardial Fat Thickness as the Marker for Diagnosis of CAD

\begin{tabular}{ll}
\hline Area under the curve (AUC) & 0.07 \\
$95 \%$ CI of AUC & $0.58-0.82$ \\
P-value & $0.002 *$ \\
Cut-off & 4.65 \\
Sensitivity & $71.6 \%$ \\
Specificity & $73.1 \%$ \\
\hline
\end{tabular}

Although its role is still unclear, echocardiographic epicardial fat has been associated with CAD. Epicardial fat thickness $>7 \mathrm{~mm}$ has been associated with subclinical atherosclerosis and CAD, but only in women. However, different and lower epicardial fat thickness cutoff values $(>4.5 \mathrm{~mm})$ have shown good sensitivity and specificity to detect low coronary flow reserve in women. The absence of men may prevent the generalization of these interesting results [21]. Echocardiographic epicardial fat thickness has been shown to predict coronary flow reserve in women with angiographically normal coronary arteries.

Hirata et al [22] tested the hypothesis that echocardiographic adipose thickness especially adipose thickness in anterior inteventricular groove (EAT-AIG), where the left descending coronary artery runs, can be a marker for the presence and severity of CAD. According to the coronary angiography, subjects were divided into two groups with (CAD group: 113 patients, $67 \pm 11$ years, 89 male) and without (non-CAD group: 50 patients, $69 \pm 11$ years, 31 male) significant coronary stenosis $(>75 \%)$. The EAT-AIG, the EAT on the right ventricular free wall (EATRV), and pericardial adipose thickness were measured by 2D echocardiography. By ROC analysis, systolic EAT-AIG thickness $>7.3 \mathrm{~mm}$ predicted the presence of significant coronary stenosis by $59 \%$ sensitivity and $78 \%$ specificity. Our study showed that epicardial fat thickness $>4.65 \mathrm{~mm}$ predicted the presence of significant CAD. Also, the patients in CAD group were of lower age group $(60.30 \pm 8.36)$ in our study as compared to past study $(67 \pm 11)$. Similarly, significant CAD was described as $>75 \%$ stenosis in any $>2.5 \mathrm{~mm}$ epicardial vessels, while in our study, we took $>70 \%(>50 \%$ in LMCA stenosis) as significant CAD.

Ding et al [23] performed a case-cohort study in the multiethnic study of atherosclerosis (MESA) cohort, investigating a random sample of 998 participants and the 147 individuals who developed coronary events. Ejection fraction was associated with CAD (RR for increase of one SD in EF of $1.26(95 \%$ 


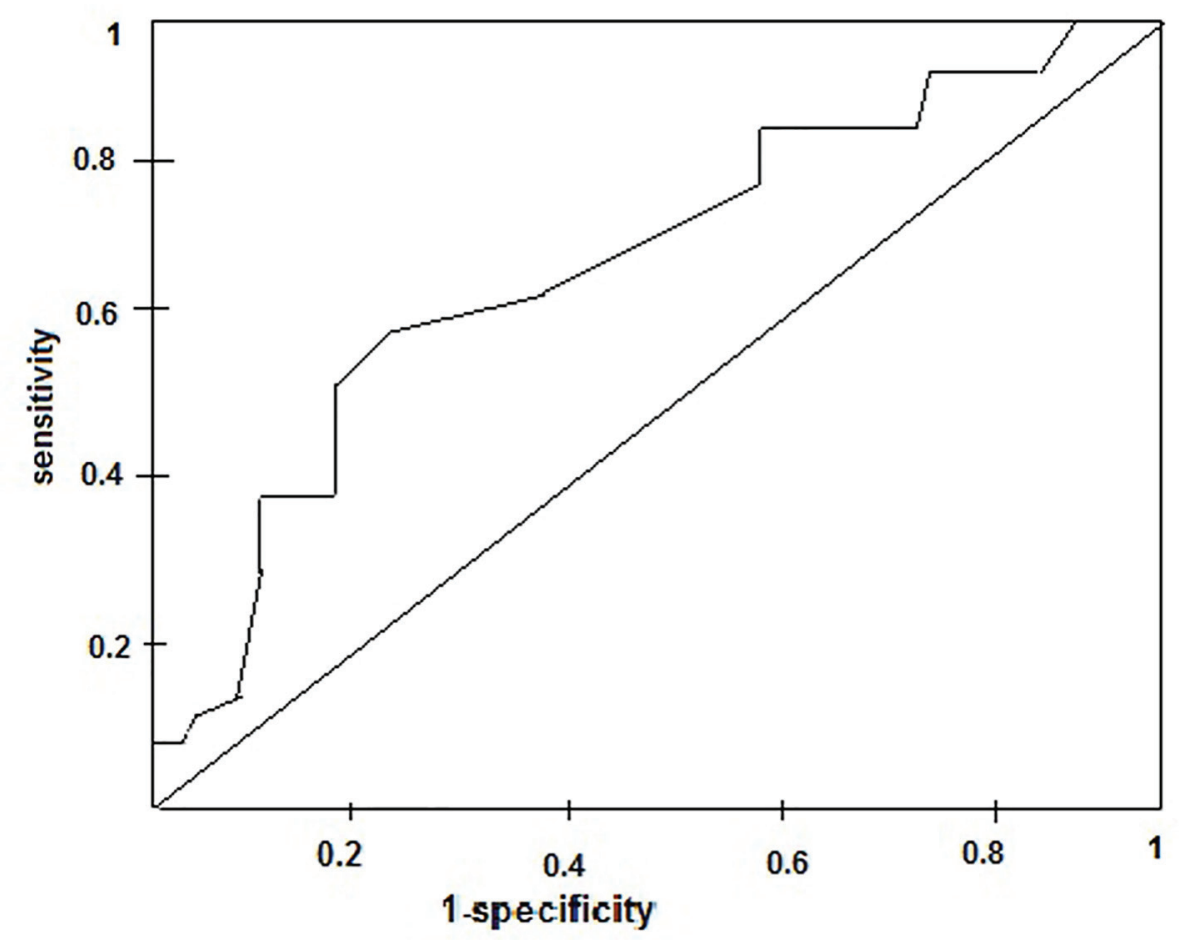

Figure 9. The area under the curve on receiver operating characteristic curve analysis of epicardial fat thickness as the marker for diagnosis of CAD.

CI: $1.01-1.59)$ ) even after adjustment for cardiovascular risk factors.

Adipose tissue usually does not deposit uniformly around the heart; it accumulates predominantly in the interventricular and atrioventricular grooves. However, adipose tissue distribution becomes rather uniform in patients with increased EAT thickness, especially in patients with abnormally large amounts of adipose tissue. We demonstrated that EAT thickness has an additive and stronger prognostic value for predicting coronary artery lesions. This parameter may help select certain patients for high dose statin therapy, intense life style modification and revascularization if needed. This simple measurement may be included in the routine echocardiographic examination.

EAT calculation by echocardiography requires very little time and can be easily applied during an examination for evaluation of morphological and functional cardiac parameters in patients with obesity, diabetes, and hypertension. However, it does require a certain experience necessary to measure EAT. In fact, EAT may produce an echo-free space that can be mistaken for pericardial fluid. Sometimes, it appears as hyperechoic space, if in large amount ( $>15 \mathrm{~mm})$. Further studies looking at the appropriate cut-off thickness of EAT and the sites of measurement to be used are needed.

\section{Limitations of using echocardiography to measure epicar- dial fat}

The study population consisted entirely of Indian patients, so the relevance of this study to patients of other ethnic backgrounds awaits further research. The non-CAD group, defined as having $<50 \%$ coronary stenosis, may have included patients with vulnerable coronary plaques who are at substantial risk for acute coronary syndromes. Echocardiography may be not the optimal technique for the quantification of epicardial fat as it is a linear measurement at single location; therefore, it may not reflect the variability of fat thickness or total epicardial fat volume. Multi-detector computed tomography is more sensitive and specific than echocardiography for measuring fat thickness in deeper epicardial fat layers. Epicardial fat volume, rather than its thickness, may in fact be the most consistent measure of risk, as recently suggested [24, 25]. However, we believe the limits of agreement are acceptable for screening purposes. Furthermore, the result of ROC analysis cannot be applied broadly to patients with suspected CAD, and the sensitivity and specificity of an EAT value for diagnosing CAD need to be confirmed in a future independent study. Further investigation in a large-scale study is warranted to confirm the clinical efficacy of measuring EAT thickness using echocardiography in patients with atherosclerosis.

\section{Conclusion}

EAT is consistently associated with CAD, although the magnitude of these associations is probably lower than previously expected. EAT measured using 2D TTE significantly correlated with the severity of coronary artery stenosis in patients with 
known CAD. According to current knowledge, EAT thicknesses $>5 \mathrm{~mm}$, or a volume $>125 \mathrm{~mL}$ or $68 \mathrm{~mL} / \mathrm{m}^{2}$ might be considered abnormal. Its assessment is sensitive, easy, non-invasive and cost-effective for prediction and severity of CAD patients.

\section{Financial Disclosures}

None.

\section{Conflict of Interest}

None.

\section{References}

1. Visscher TL, Seidell JC, Molarius A, van der Kuip D, Hofman A, Witteman JC. A comparison of body mass index, waist-hip ratio and waist circumference as predictors of all-cause mortality among the elderly: the Rotterdam study. Int J Obes Relat Metab Disord. 2001;25(11):17301735 .

2. Hubert HB, Feinleib M, McNamara PM, Castelli WP. Obesity as an independent risk factor for cardiovascular disease: a 26-year follow-up of participants in the Framingham Heart Study. Circulation. 1983;67(5):968977.

3. Manson JE, Colditz GA, Stampfer MJ, Willett WC, Rosner B, Monson RR, Speizer FE, et al. A prospective study of obesity and risk of coronary heart disease in women. $\mathrm{N}$ Engl J Med. 1990;322(13):882-889.

4. Kaplan NM. The deadly quartet. Upper-body obesity, glucose intolerance, hypertriglyceridemia, and hypertension. Arch Intern Med. 1989;149(7):1514-1520.

5. Washio M, Hayashi R. Past history of obesity (overweight by WHO criteria) is associated with an increased risk of nonfatal acute myocardial infarction: a case-control study in Japan. Circ J. 2004;68(1):41-46.

6. Peiris AN, Sothmann MS, Hoffmann RG, Hennes MI, Wilson CR, Gustafson AB, Kissebah AH. Adiposity, fat distribution, and cardiovascular risk. Ann Intern Med. 1989;110(11):867-872.

7. Corradi D, Maestri R, Callegari S, Pastori P, Goldoni M, Luong TV, Bordi C. The ventricular epicardial fat is related to the myocardial mass in normal, ischemic and hypertrophic hearts. Cardiovasc Pathol. 2004;13(6):313-316.

8. Yudkin JS, Eringa E, Stehouwer CD. "Vasocrine" signalling from perivascular fat: a mechanism linking insulin resistance to vascular disease. Lancet. 2005;365(9473):1817-1820.

9. Sacks HS, Fain JN. Human epicardial adipose tissue: a review. Am Heart J. 2007;153(6):907-917.

10. Lim S, Meigs JB. Ectopic fat and cardiometabolic and vascular risk. Int J Cardiol. 2013;169(3):166-176.

11. Bambace C, Telesca M, Zoico E, Sepe A, Olioso D, Rossi A, Corzato F, et al. Adiponectin gene expression and adipocyte diameter: a comparison between epicardial and subcutaneous adipose tissue in men. Cardiovasc Pathol. 2011;20(5):e153-156.

12. Mazurek T, Zhang L, Zalewski A, Mannion JD, Diehl JT, Arafat H, Sarov-Blat L, et al. Human epicardial adipose tissue is a source of inflammatory mediators. Circulation. 2003;108(20):2460-2466.

13. Iacobellis G, Corradi D, Sharma AM. Epicardial adipose tissue: anatomic, biomolecular and clinical relationships with the heart. Nat Clin Pract Cardiovasc Med. 2005;2(10):536-543.

14. Iacobellis G. Epicardial and pericardial fat: close, but very different. Obesity (Silver Spring). 2009;17(4):625; author reply 626-627.

15. Tansey DK, Aly Z, Sheppard MN. Fat in the right ventricle of the normal heart. Histopathology. 2005;46(1):98-104.

16. Gorter PM, van Lindert AS, de Vos AM, Meijs MF, van der Graaf Y, Doevendans PA, Prokop M, et al. Quantification of epicardial and peri-coronary fat using cardiac computed tomography; reproducibility and relation with obesity and metabolic syndrome in patients suspected of coronary artery disease. Atherosclerosis. 2008;197(2):896-903.

17. Jeong JW, Jeong MH, Yun KH, Oh SK, Park EM, Kim YK, Rhee SJ, et al. Echocardiographic epicardial fat thickness and coronary artery disease. Circ J. 2007;71(4):536-539.

18. Iacobellis G, Gao YJ, Sharma AM. Do cardiac and perivascular adipose tissue play a role in atherosclerosis? Curr Diab Rep. 2008;8(1):20-24.

19. Iacobellis $\mathrm{G}$, Barbaro $\mathrm{G}$. The double role of epicardial adipose tissue as pro- and anti-inflammatory organ. Horm Metab Res. 2008;40(7):442-445.

20. Chaowalit N, Somers VK, Pellikka PA, Rihal CS, LopezJimenez F. Subepicardial adipose tissue and the presence and severity of coronary artery disease. Atherosclerosis. 2006;186(2):354-359.

21. Park SH, Shim KW. Reduction in visceral adiposity is highly related to improvement in vascular endothelial dysfunction among obese women: an assessment of endothelial function by radial artery pulse wave analysis. Yonsei Med J. 2005;46(4):511-518.

22. Hirata Y, Yamada H, Kusunose $K$, Iwase T, Nishio S, Hayashi S, Bando M, et al. Clinical Utility of Measuring Epicardial Adipose Tissue Thickness with Echocardiography Using a High-Frequency Linear Probe in Patients with Coronary Artery Disease. J Am Soc Echocardiogr. 2015;28(10):1240-1246 e1241.

23. Ding J, Hsu FC, Harris TB, Liu Y, Kritchevsky SB, Szklo M, Ouyang $\mathrm{P}$, et al. The association of pericardial fat with incident coronary heart disease: the Multi-Ethnic Study of Atherosclerosis (MESA). Am J Clin Nutr. 2009;90(3):499-504.

24. Alexopoulos N, McLean DS, Janik M, Arepalli CD, Stillman AE, Raggi P. Epicardial adipose tissue and coronary artery plaque characteristics. Atherosclerosis. 2010;210(1):150-154.

25. Mahabadi AA, Berg MH, Lehmann N, Kalsch H, Bauer M, Kara K, Dragano N, et al. Association of epicardial fat with cardiovascular risk factors and incident myocardial infarction in the general population: the Heinz Nixdorf Recall Study. J Am Coll Cardiol. 2013;61(13):1388-1395. 